\title{
Tratamiento del cáncer de colon estadios II, III y IV
}

\author{
E. Aranda Aguilar
}

El cáncer colorrectal ocupa el tercer lugar en frecuencia en el hombre y el segundo en la mujer. Es la cuarta causa de mortalidad por cáncer en los países occidentales, por lo que representa un importante problema de salud pública. Si tenemos en cuenta que más del $50 \%$ de los pacientes con cáncer colorrectal tendrán enfermedad metastásica o localmente avanzada irresecable en algún momento de la evolución de la enfermedad, el tratamiento sistémico adquiere un importante papel en la estrategia terapéutica de esta neoplasia.

\section{Tratamiento del cáncer de colon estadio IV}

\section{Fluorouracilo}

Tras el empleo de múltiples fármacos y combinaciones, el 5-Fluorouracilo se situó en la década de los 70 como el fármaco más activo, y hoy día, tras haber potenciado su eficacia con la modulación bioquímica y con la infusión continua, sigue siendo en combinación, el citostático de elección en primera línea de tratamiento. Ya no es discutible el beneficio de la quimioterapia en el cáncer de colon metastático o avanzado. El dilema actual estriba en decidir cuál es el mejor tratamiento quimioterápico que puede recibir un paciente en base a las características que presenta, ya sea inherente a la neoplasia o al propio paciente.

Un mejor conocimiento de la biología de la célula tumoral, ha permitido el desarrollo de nuevos citotóxicos activos en el carcinoma colorrectal, con algunos de ellos con mecanismos de acción similar al 5FU (raltitrexed y capecitabina) y otros con mecanismos completamente diferentes (oxaliplatino e irinotecan), lo que está permitiendo en estos últimos años combinarlos con un espectro de mayor toxicidad, pero con la esperanza de aumentar la tasa de respuestas y sobre todo aumentar la calidad de vida y la supervivencia de nuestros pacientes.

Servicio Oncología Médica

Hospital Reina Sofía

Córdoba
La combinación de citotóxicos es hoy día un concepto que parece incuestionable y necesario en el tratamiento del cáncer, si queremos obtener una mayor capacidad antitumoral con la mínima toxicidad y evitar en la medida de lo posible la formación de resistencias.

\section{Irinotecan}

El irinotecan es un fármaco inhibidor de la enzima topoisomerasa 1 que ha demostrado actividad tanto en primera y segunda línea de tratamiento del carcinoma de colon, con una tasa de respuesta global de un $17-25 \%$, equiparable a las obtenidas por el 5FU modulado.

Los resultados de los estudios fase II realizados hasta el momento son bastante esperanzadores, obteniéndose tasas de respuestas objetivas en segunda y tercera línea de tratamiento que van desde el 15 al $30 \%$.

Dos estudios fase III han sido definitivos para establecer la asociación de 5-Fluorouracilo e Irinotecan como el esquema de elección en la primera línea de tratamiento del carcinoma colorrectal avanzado. En el primero de ellos (Dovillart y col.) se han randomizado 338 pacientes ha recibir un esquema con CPT-11 más 5FU infusión continua modulado con ácido folínico, frente al mismo esquema sin CPT-11. El tratamiento se mantiene hasta progresión. Este estudio demuestra que la combinación con CPT-11 supone un incremento estadísticamente significativo en la tasa de respuestas ( $R$. Globales: $41 \%$ vs $23 \%$ ), en el tiempo a la progresión 6,7 meses vs 4,4 meses y también en la supervivencia $(17,4$ meses vs 14.1 meses). El otro estudio (Saltz y col.) randomiza 457 pacientes a recibir 5-Fluorouracilo bolus más Lederfolin y CPT- 11 frente al mismo esquema sin CPT-11, obteniendo de manera significativa mejores tasas de respuesta $39 \%$ vs $21 \%$, mejor tiempo a la progresión (7 meses vs 4,3 meses) y mejor supervivencia ( 14 meses vs 12.9 meses).

\section{Oxaliplatino}

El Oxaliplatino (L-OHP) es un análogo del cisplatino pero con patrones de actividad y toxicidad diferentes. En monoterapia es un fármaco que ha demostrado una actividad similar al 5-FU, con un porcentaje de respuestas objetivas (sin tener en cuenta estabilizaciones) entre el 17-22\%. Su mecanismo de acción es diferente del 5FU, no habiéndose demostrado resistencia cruzada; si además tenemos en cuenta que la 
toxicidad no es superponible y que in vitro se ha demostrado un sinergismo, el oxaliplatino es un fármaco muy interesante para la combinación con 5-FU.

Las respuestas obtenidas van desde el 25 al 58\%, con una tasa de respuestas completas del 3,5 al 5\%. De forma global, el tiempo libre de progresión de 5,8 meses a 11 meses y la supervivencia de 12 a 17 meses; la tasa de respuestas es similar en las diferentes localizaciones metastásicas.

Una cuestión fundamental fue conocer si la adición de oxaliplatino incrementaba de forma significativa la tasa de respuestas y la supervivencia con respecto a las que se obtiene con tratamientos con 5FU. Con este objetivo se han realizado dos estudios europeos Fase III. En un primer estudio (Giachetti y col.) se compara un régimen de administración cronomodulada de oxaliplatino más 5FU y ácido folínico con un esquema sin oxaliplatino. Tras inclusión de 100 pacientes con carcinoma colorrectal metastásico no pretratados en cada rama, la tasa de respuestas (53\% vs. $16 \%$ ) y el tiempo a la progresión ( 8,7 vs 6,7 meses) fue significativamente mayor en la rama del oxaliplatino; en cuanto a la supervivencia no hubo diferencias (19 vs. 19,9 meses).

En el segundo fase III realizado (De Gramont y col.), se compara un esquema bisemanal que incluye oxaliplatino, $5 F U$ bolus modulado y 5FU en infusión continua, con el mismo esquema $\sin$ oxaliplatino. Incluidos 420 pacientes, de nuevo se confirma una mayor tasa de respuestas $(50,7 \%$ vs. $22,3 \% ; p<0,05$ ) e intervalo libre de progresión (9 vs. 6,2 meses), a favor de la rama con oxaliplatino.

En este mismo sentido recientemente en ASCO de 2002 Grothey y col. han presentado un estudio fase III en el que comparan FUFOX con 5-FU+LV (régimen de la Mayo) demostrando con la inclusión de 242 pacientes que FUFOX es más efectivo $(48,3 \%$ vs $22,6 \% \mathrm{p}<0.0001)$, tiene mejor tiempo a la progresión (5,3 meses vs 7,9 meses $p<0.0001)$, mejor supervivencia $(16,1$ meses vs 20,4 meses) y es menos tóxico.

Golberg y col., también en ASCO de 2002, han presentado los resultados de un estudio fase III (N9741) que si bien se inició con 5 ramas, al final compara IFL frente a Folfox encontrándose una mayor eficacia a favor del Folfox $138 \%$ vs $29 \% p<0.03$ ), mejor tiempo a la progresión $p<0.0009$ y una mejor supervivencia $(14,1$ meses vs 18,6 meses $\mathrm{p}<0.002$ ), fase III que como los mismos autores discuten ha comparado 5-FU en ic (Folfox) frente a bolus (IFL) y la influencia del rescate en la supervivencia; esto nos muestra el beneficio de los regímenes combinados utilizando oxaliplatino, beneficio ya conocido en gran parte de Europa.

\section{Capecitabina}

La Capecitabina es un análogo del 5FU diseñado con el objetivo de disminuir la toxicidad digestiva e incrementar la actividad. Tras su absorción, es metabolizada a nivel hepático y activada por la timidin fosforilasa presente en mayor concentración en las células tumorales. Su mayor desarrollo clínico se ha producido en el tratamiento del carcinoma de mama. In vivo ha demostrado sinergismo cuando se combina con ciclofosfamida, taxanos y mitomicina. En los últimos años también se está desarrollando en el tratamiento del carcinoma colorrectal. Estudios fase III han demostrado una eficacia superior a esquemas clásicos de 5 Fu bolus modulado.

Se han realizado estudios fase I y II tanto con capecitabi- na más oxaliplatino (Evans y col.), así como con Irinotecan (Twelves y col.), demostrando su factibilidad y actividad, por lo que podría ser una alternativa a la infusión continua de 5Fluorouracilo. Se ha observado una eficacia similar con ambas combinaciones, aunque los resultados son prematuros (37,5\% respuesta Xeloda+CPT- 11 vs $41.2 \%$ Xeloda+Oxaliplatino) (Jordan et al).

Actualmente se han puesto en marcha dos estudios, uno internacional con capecitabina más CPT-11 vs. 5-Fluorouracilo infusión continua más CPT-11 y otro en el seno del grupo TTD que compara capecitabina más oxaliplatino más 5Fluorouracilo más oxaliplatino con el objeto de conocer si realmente la capecitabina desplazará al Fluorouracilo o no.

En otro sentido, debemos tener en cuenta el mayor conocimiento de la biología molecular que nos está permitiendo conocer factores biológicos que nos pueden predecir el pronóstico, así como la respuesta y toxicidad al tratamiento que vamos a utilizar, (TS, TP, DPD, P53, MSI, etc), lo que nos va a permitir, en un futuro no muy lejano, adecuar el tratamiento que vamos a utilizar a las características moleculares de la célula/as tumorales que tiene cada paciente.

Al mismo tiempo se ha profundizado en la fisiopatología de la célula tumoral y conocemos cómo hay una autosuficiencia en las señales de crecimiento, una insensibilidad a las señales inhibitorias del crecimiento, una alteración de la muerte celular programada, una capacidad de replicación ilimitada, una angiogénesis mantenida, así como la capacidad de invadir los tejidos y dar metástasis a distancia. Estos conocimientos han hecho posible la detección de nuevas dianas terapéuticas y el diseño de fármacos, fundamentalmente citostáticos, que van a actuar en las diferentes dianas, de manera que emerge con fuerza la terapia génica como tratamiento en el cáncer intentando impedir la expresión de los oncogenes, utilizando la p53 como diana terapéutica, aumentando la inmunogenicidad tumoral, utilizando vectores que porten genes suicidas, diseñando terapias antiangiogénicas y sobre todo diseñando terapias combinadas y nuevas estrategias terapéuticas que nos permitirán, sin lugar a dudas, seguir avanzando en el tratamiento del cáncer.

En definitiva, se ha pasado en poco tiempo de un tumor que clásicamente era considerado quimiorresistente, a un tumor que podemos denominar quimiosensible. Con el avance terapéutico de los últimos años se ha conseguido aumentar el tiempo a la progresión o la supervivencia de nuestros pacientes, así como la calidad de vida de los mismos. El tratamiento quimioterápico que se propugna como estándar para el cáncer colorrectal metastásico es la poliquimioterapia, basada en combinaciones de 5FU en infusión continua más o menos lederfolin y bien Irinotecan bien oxaliplatino. Permanecemos en espera de conocer si la capecitabina podrá sustituir al 5FU en los esquemas de combinación lo que daría, manteniendo la eficacia, más comodidad para el paciente.

\section{Tratamiento del cáncer de colon estadio III}

Los resultados del tratamiento complementario del cáncer de colon ha sido considerado uno de los avances más importantes en clínica oncológica en la última década, por el impacto socio-sanitario que ha tenido en el aumento de curaciones debido a la incidencia de este tumor.

El Intergrupo, en 1990 (Moertel et al), comunicaron un es- 


\section{E. Aranda Aguilar}

tudio en el que se obtenía beneficio en cuanto a la supervivencia y reducción de riesgo de recaída en aquellos pacientes que recibían tratamiento. El estudio mostró un $15 \%$ de reducción absoluta o un $40 \%$ de reducción relativa en el riesgo de recidiva y un $33 \%$ de reducción en el riego de muerte.

Al mismo tiempo, la combinación modulada de 5FU+Lederfolin(LV) también demostró en diferentes estudios ser superior a la cirugía sola: en el estudio del NSABP el intervalo libre de enfermedad (73 vs 64\%) y la supervivencia (84 vs $77 \%$ ) fue superior a favor de la modulación frente al MOF. En el estudio del IMPACT también demostró con 5FU+LV a los tres años una reducción del $22 \%$ de mortalidad.

Más recientemente, se han realizado tres estudios americanos, donde se han incluido miles de pacientes y en los que han participado la mayoría de grupos americanos (NCCTG, $\mathrm{NCIC}$, Intergrupo 0089, NSABB C-04) intentando demostrar si el 5FU+LV era igual o no al 5FU+ Levamisole y en ese caso cuál sería el tratamiento complementario estándar para el cáncer de colon estadio III. Al final se reconoció como estandar al $5 \mathrm{FU}+\mathrm{LV}$.

En USA se propusieron dos regímenes como estándar: el del Roswell Park y el de la Clínica Mayo; en Europa fue este último el que se ha usado en la mayoría de los centros. Consiste en LV $20 \mathrm{mg} / \mathrm{m}^{2}+5 \mathrm{FU} 425 \mathrm{mg} / \mathrm{m}^{2}$ dia $1-5$ cada 28 días y durante 6 meses.

Al haber demostrado que la utilización de regimenes de $5 \mathrm{FU}$ en IC era superior a los esquemas bolus, así como que la poliquimioterapia con los nuevos fármacos (Irinotecan u Oxaliplatino) en cáncer de colon metastásico se iniciaron varios ensayos clínicos (MOSAIC, V307) con el objetivo de demostrar que también la poliquimioterapia con esquemas de 5FU + Irinotecan u Oxaliplatino eran superiores al estandar la Clínica Mayo, en este sentido en el último congreso americano se presentaron los datos del MOSAIC en el que se demuestra que en cuanto a tiempo libre de enfermedad el esquema de $5 \mathrm{FU}+\mathrm{LV}+\mathrm{Oxaliplatino} \mathrm{es} \mathrm{superior} \mathrm{al} \mathrm{mismo} \mathrm{esquema} \mathrm{sin} \mathrm{oxa-}$ liplatino, estamos a la espera de datos de supervivencia.

\section{Tratamiento en el cáncer de colon estadio II}

La cuestión de si el estadio II del cáncer de colon se beneficia con la quimioterapia adyuvante permanece controvertida. Esquemáticamente podemos dividir los datos existentes en la literatura como datos a favor y datos en contra.

Pro

Contra

\begin{tabular}{ll}
\hline Metaanálisis NSABP & Meta-análisis IMPACT \\
Gill (ASCO 2003) & SEER-Medicare (Scharg) \\
NACCP trial & INT 0035 trial
\end{tabular}

En el INT-0035, se comparó 5-FU más Levamisole versus cirugía sola que muestra una similar reducción del porcentaje de recidivas (32\%) en estadio II igual que en estadio III, no mostró beneficio para la supervivencia, probablemente por el escaso poder del estudio.

El IMPACT B2 analiza 5 trabajos en los cuales los pacientes fueron randomizados después de la intervención quirúrgica para recibir 5-FU+LV o nada, concluyendo que no es recomendable para el estadio B2 utilizar de rutina el trata- miento complementario con $5-\mathrm{FU}+\mathrm{LV}$. A los 5 años la supervivencia fue de un $80 \%$ vs $82 \%$ y el porcentaje libre de enfermedad de 73 y $76 \%$ para control y tratamiento, respectivamente.

Por otro lado, el grupo NSABP combinando datos de cuatro trabajos que incluín estadios $B$ y $C$, concluyen que los pacientes con cáncer de colon estadio $B$ se benefician de la quimioterapia complementaria. La reducción relativa de mortalidad, recidiva o intervalo libre de enfermedad fue tanto para estadio B como para el C. La reducción de mortalidad fue del $30 \%$ para pacientes con cáncer de colon estadio $\mathrm{B}$, independientemente de la presencia o no de factores pronósticos desfavorables.

Es posible que en la situación actual tan sólo un subgrupo de pacientes puedan beneficiarse del tratamiento adyuvante, - que este beneficio sea muy limitado, obteniendo beneficio tan sólo 2 o 3 pacientes de cada 100 tratados. De cualquier manera, deberemos estar atentos a los resultados pendientes de los grandes fases III pendientes que nos permitirán conocer cuál es el papel de las combinaciones de 5-FU+/- LV con Irinotecan u Oxaliplatino (V-307; MOSAIC, etc.); respecto al MOSAIC ya en el último congreso americano se presentaron datos de beneficio para el intervalo libre de enfermedad tanto para el estadio III como para el II. Al mismo tiempo están siendo investigados el papel de las fluoropirimidinas orales (Capecitabina y UFT) en el tratamiento adyuvante del cáncer de colon.

La identificación de subgrupos de pacientes con cáncer de colon en estadio II con mal pronóstico y que puedan beneficiarse de un tratamiento complementario es una prioridad absoluta para el futuro. La contribución de la biología molecular y la genética no ha sido suficiente, hasta el momento, para que puedan ser introducidos en la práctica clínica para poder decidir qué pacientes con cáncer de colon estadio II tratar y cuáles no.

Mientras llegan los resultados que nos permitan conocer mejor el pronóstico del paciente al que tenemos que tratar, en la práctica clínica cotidiana tendremos que seguir haciendo la evaluación del paciente en base a los datos clínicos y anatomopatológicos clásicos, estableciendo una clara y franca discusión de los pros y contras de un tratamiento adyuvante, con cada paciente que haya sido operado de un cáncer de colon ganglios negativos.

\section{Bibliografía}

Meta-analisis. The Meta-analysis Group in Cancer: Efficacy of intravenous infusion of fluoruracil compared with bolus administration in advanced colorectal cancer. J. Clin. Oncol. 1998; 16:391-408.

Giacchetti S, Itzhaki M, Gruia et al. Long-term survival of patients with unresectable colorectal cancer liver metatases following infusional chemotherapy with 5-fluouracil, leucovorin, oxaliplatino and surgery. Ann Oncol 1999; 10:663-9.

Douillart JY, Cunninham D, Roth $A D$ et al. Irinotecan combined with fluorouracil compared with fluorouracil alone as first line treatment for metastatic colorectal cancer: a multicentre randomised trial. The Lancet 2000; 355:9209.

Saltz LB, Cox JV, Blanke C y cols. Irinotecan plus Fluorouracil and leucovorin for metastatic colorectal cancer. New Eng Med. 2000; 343;13:905-14. 
Giaccheti S, Perpoint B, Zidani R y cols. Phase III Multicentre Randomized Trial of Oxaliplatin Added to Chronomodulated Fluorouracil-Leucovorin as First line treatment of metastatic colorectal cancer. J Clin Oncol 2000; 18:136-47.

De Gramont A, Figer A, Seymor My cols. A randomized trial of leucovorin and 5-Fluorouracil with or without oxaliplatin in advanced colorectal cancer. J Clin Oncol 2000; 18:2938-47.

Twelves C, Boyer M, Findlay $M$ et al. Capecitabina (XelodaTM) improves medical resources use compared with 5-Fluorouracil plus leucovorin in a phase III trial conducted in patient with advanced colorectal cancer. Eur J Cancer 2001; 37:597-604.

Tournigand C, Louvet C, Quinaux E, et al. FOLFIRI Followed by FOLFOX Folowed by FOLFIR in Metastatic Colorectal Cancer (MCRC): Final Results of a Phase III Study. 2001. Procc ASCO 20:494.

Grothey A, Deschler B, Krening $\mathrm{H}$ et al. Phase III study of bolus 5fluorouracil (5-FU)/ folonic acid (FA) (Mayo) vs weekly high-dose 24h 5-FU infusional/ FA+ Oxaliplatin (OXA) (FUFOX) in advanced colorectal cancer. 2002. Procc ASCO 21:512.

Golberg RM, Morton RF, Sargent DJ et al. N9741: oxaliplatino (oxal) or CPT-1 1+5-fluorouracil (5FU)/leucovorin (LV) or oxal+CPT- 11 in advanced colorectal cancer (CRC). Initial toxicity and response data from a GI Intergroup study. 2002. Procc ASCO 21:511.

Jordan K, Grothey A, Kellner O et al. Randomized phase II trial of capecitabina plus irinitecan vs capecitabina plus oxaliplatino as first-line therapy in advanced colorectal cancer (ACRC): results of an interin analysis. 2002. Procc ASCO 21:2225.

Salonga D, Danenberg M, Metzger $R$ et al. Colorecta tumors responding to 5-Fluorouracil have low gene expression levels of dihidropyrimidine dehidrogenase, thymidilate synthasa, and thymidine phosphoriyase. Clinical Cancer Research 2000; 6:1322-7.

Stoehlmacher J, Ghaderi V, lobal S et al. A polymorphism of the XRCCl gene predicts for response to platinum based treatment in advanced colorectal cancer. Anticancer Res 2001; 21:30759.

Moertel CG, Fleming TR, Macdonald JS, Haller DG, Laurie JA, Goodman PJ, Ungerleider JS, Emerson WA, Tormey DC, Glick JH. Levamisole and fluorouracil for adjuvant therapy of resected colon carcinoma. N Engl J Med 1990: 322: 352-8.

Wolmark N, Rockette H, Fisher B, Wickerman DL, Redmond C, Fisher ER, Jones J, Mamounas EP, Ore L, Petrelli NJ. The benefit of leucovorin-modulated fluorouracil as postoperative adjuvant therapy for primary colon cancer. Results form NSABP protocol C03. J Clin Oncol 1993; 11:1879-87.
International Multicenter Pooled Analysis of Colorectal Cancer Trials (IMPACT). Efficacy of adjuvant fluoracil and folinic acid in colon cancer. Lancet 1995; 345: 939-44.

Moore HCF, Haller DG. Adjuvant therapy of colon cancer. Sem Oncol 1999; 26:545-55.

Liefers GJ, Cleton-Jansen AM, van de Velde CJ, Hermans J, van Krieken JH, Cornelisse CJ, Tollenaar RA. Micrometastases and survival in stage II colorectal cancer. N Engl J Med 1998; 339:223-228.

Gryfe R, Kim H, Hsieh ETK, Aronson MD, Holowaty EJ, Bull SB, Redston M, Gallinger S. Tumor microsatellite instability and clinical outcome in young patients with colorectal cancer. N Engl J Med 2000; 342:69-77.

Elsaleh H, Joseph D, Grieu F, Zeps N, Spry N, Lacopetta B. Association of tumor site and sex with survival benefit from adjuvant chemotherapy in colorectal cancer. The Lancet 2000; 335:1745-50.

Moertel CG, Flemung TR, Macdonald JS, Haller DG, Laurie JA, Tangen CM, Ungerleider JS, Emerson WA, Torney DC, Glick JH, Veeder MH, Mailliard JA for the North Central Cancer Treatment group, Southwest Oncology Group, and Eastern Cooperative Oncology Group. Intergroup study of fluorouracil plus levamisole as adjuvant therapy for stage II/Dukes'B2 colon cancer. J Clin Oncol 1995; 13:2936-43.

International Multicenter Pooled Analysis of BS Colon Cancer Trials (IMPACT B2) Investigators. Efficacy of adjuvant fluorouracil and folinic acid in B2 colon cancer.. J Clin Oncol 1999; 17:135663.

Mamounas E, Wieand S, Wolmark N, Bear HD, Atkins JN, Song K, Jones J, Rockette H. Comparative efficacy of adjuvant chemotherapy in patients with Dukes'B versus Dukes' $C$ colon cancer. Results from four national surgical adjuvant breast and bowel project adjuvant studies (C-01, C-02, C-03, and C-04). J Clin Oncol 1999; 17:1349-55.

Scharg D. Defining optimal treatment for stage II colon cancer: Does decision analysis help?. Gastroenterology 1999; 117:1005-8.

Michel P, Merle V, Chiron A, Ducrotte P, Paillot B, Hecketsweiler P, Czernichow P, Colin R. Postoperative management of stage II/III colon cancer. A decision analysis. Gastroenterology 1999; 117:784-93.

Gill S, Loprinzi CL, Sargent DJ et al.: Using a pooled analysis to improve the understaging of adjuvant therapy benefit for colon cancer. Proc Am Soc Clin Oncol 2003; 22:1014.

De Gramont A, Banzi M, Navarro M et al.: Oxaliplatin/5-FU/LV in adjuvant colon cancer: Results of the International randomized mosaic trial. Proc Am Soc Clin Oncol 2003; 22:1015. 